\title{
Data delivery techniques in content centric routing for IoT: a systematic review
}

\author{
Pooja Patel $^{{ }^{*}}$, Hiren B. Patel ${ }^{2}$ and Bela Shrimali ${ }^{3}$ \\ M.Tech Student, Department of Computer Science, LDRP Institute of Technology, Affiliated Under Kadi Sarva \\ Vidyalaya, India ${ }^{1}$ \\ Professor and HOD, Department of Computer Science, LDRP-Institute of Technology and Research, Gandhinagar, \\ India $^{2}$ \\ Assistant Professor, Department of Computer Science, LDRP-Institute of Technology and Research, Gandhinagar, \\ India $^{3}$
}

C2018 ACCENTS

\begin{abstract}
Internet of Things (IoT) connects a number of devices that share data through the internet. Communication between these devices causes a large amount of data in the network that creates a lot of traffic. In order to manage the traffic, the concept of content-centric networking $(C C N)$ is introduced and identified as an effective network model for efficient data delivery in a wireless sensor network. CCN determines routing path based on the content. In this paper, we discuss the CCN architecture and its packet forwarding mechanisms. Also, we provide a review of different techniques of CCN covering naming schemes, architecture and the parameters evaluated and discussed in recent state-of-an-art. This review analysis may help the new researchers to go in-depth in the domain of CCN.
\end{abstract}

\section{Keywords}

IoT, CCN, Naming, Architecture, Message, Routing.

\section{Introduction}

IoT is a network of communication in which physical devices are connected to each other through the internet where they share data that can be accessed anywhere at any time [1]. Wireless sensors networks (WSNs) are considered to be the most important element of the IoT. It plays an important role to support the communication between user devices and large-scale resource-constrained devices such as sensors and other smart devices. Some of the applications such as smart building monitoring, healthcare, environmental monitoring, production control, security and surveillance applications. Traditional routing protocols aimed to support host to host communications among nodes that are distinguished by an IP address fails in a distributed wireless network as a result of frequently changing network topology generated through mobility of node and regular link failure. Moreover, IoTs comprises of heterogeneous devices and most of these devices are small, low power with a limited memory, low cost, and constraint-oriented wireless sensors.

*Author for correspondence

445
Data is not available most of the time, due to low memory and low battery lifetime.

To overcome the problem, the CCN paradigm is used which rely on content-based forwarding and innetwork caching for sending the packet data in the wireless network, which significantly saves the energy and bandwidth, reduces network latency and extends the network lifetime in a resourceconstrained IoT network environment. The main features of $\mathrm{CCN}$ in the wireless environment are discussed below:

First, user mobility is inherently maintained in CCN: when a user moves from one place to another, it issues any Interest that is not satisfied again from the new location. Provider mobility also needs updates of the routing table, but $\mathrm{CCN}$ intrinsically promotes multi-sourcing the content [2]. In CCN, mobility can be provided using Publish-Subscribe model, through which any mobile node subscribes the network for the information it needs and the publisher advertises the availability of information to the network [3]. Hence, the CCN Mobility support can play an important role in vehicular ad-hoc network (VANET) and mobile ad-hoc network (MANET) applications. 
A second important feature of $\mathrm{CCN}$ is in-network caching, which reduces the network load while caching the data to the intermediate nodes. And thus, cache enabled IoT applications can improve network performance.

Another is receiver driven communication, where $\mathrm{CCN}$ data retrieval is receiver driven and the communication only starts when a consumer wants. To get some data, a receiver places a request and the network finds the best possible source for the requested information. There are two approaches, through which a receiver can receive information: (i) coupled approach and (ii) decoupled approach. In a coupled approach, name resolution and routing are aggregated and the data is routed following a reverse path towards the consumer. The name-based routing (NBR) is involved with this approach for the content discovery. While in the decoupled approach, the Name Resolution and routing are performed independently and any path other than the request message is utilized to forward the response to the consumer. A Lookup based resolution system (LRS) can be used for the implementation of such an approach.

The fourth advantage is that $\mathrm{CCN}$ deals with alternating, dynamic topologies and short-lived connectivity in wireless network environments.

Other evolving applications are associated with exchanging of surveillance information and updates in the software of the mobile user in MANET. In the same way, in VANETs, the traffic information and climate information required by vehicles for a specified range, irrespective of their identity or IP addresses. Different scientific and military applications constructed on data gathering and data distribution in a wide-scale monitoring wireless sensor networks, that can help in naming content and the Interest/Data exchange in CCN.

This paper focuses on the $\mathrm{CCN}$ model, and it gives a complete outline and a survey of the applicability of this model in a wireless network. The rest of the paper is structured as follows: Section 2 covers CCN overview in which $\mathrm{CCN}$ integration with routing protocol for low-power and lossy networks (RPL) and architecture with the routing and forwarding mechanisms is discussed. In section 3 we present the discussion on data delivery with content centric routing (CCR) in IoT. Section 4 discusses about the challenges and research solutions in brief and Section 5 concludes the paper.

\section{CCN overview}

\subsection{CCN integration in RPL}

The integration of CCR components in the RPL protocol is described with the complete system architecture as shown in Figure 1. The integration of CCN communication layer in RPL requires a Named Data to design and implement the protocol based on CCN. For the integration of this communication layer in RPL, the Contiki operating system is used which mainly deals with the embedded system and WSNs. The communication layer in CCN comprises of a stack and a driver, which meets the implementation style of Contiki. The CCN processing, forwarding and caching functions can be implemented using Stack, while the message exchange in the lower layer is handled through drivers.

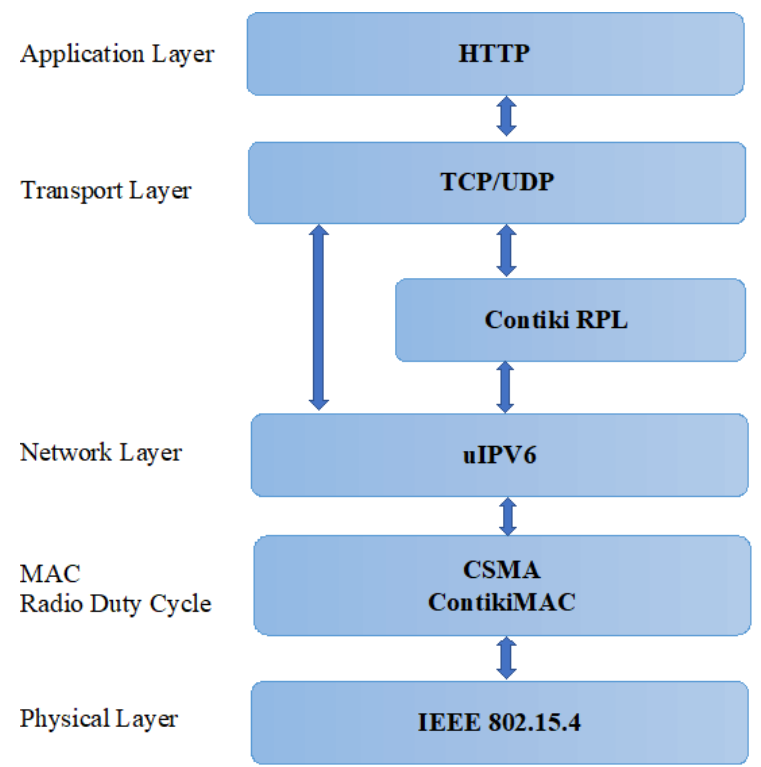

Figure 1 CCN integration into Contiki RPL [4]

The communication layer in $\mathrm{CCN}$ directly uses the MAC layer already existing in the Contiki to transfer its data messages, and hence it handles the packet transmission to deliver messages which does not depend on other transport protocol.

\subsection{CCN architecture}

The CCN architecture is formed on named data in which the content request is identified through names of the content and not with their location. The CCN method tends to the problems constraining the existing utilization of systems by expanding the accessibility of information. To reduce the traffic congestion, it provides in-network caching. While in terms of security, CCN proposes that trust based on content is simply lost when we depend on the 
location of the data. Rather than which, it incorporates the security with the network at the data level. Moreover, the location and content have no mapping, as the communication depends only on data names. So, it is simple to configure the network devices. The $\mathrm{CCN}$ protocol is used as a transport protocol in CCN network architecture.

\subsection{CCN messages}

\subsubsection{Message types}

There are two types of $\mathrm{CCN}$ messages :(i) Interest Message (IM) and (ii) Content Object (CO) message. The IM is a request of the named data. It contains the full name that identifies a piece of content. Interest packet may also contain a prefix of the content name as shown in Figure 2. A possible reply to the Interest can be a prefix of the Interest name that matches the content.

The CO message is utilized to provide data and it comprises of a data headed by the content name.

2.3.2Message formats

The physical and MAC layer uses IEEE 802.15.4 standards for implementation in Contiki which is integrated with the $\mathrm{CCN}$ communication layer. The frame size of an 802.15.4 is restricted to 127 bytes. And a payload of 72-116 bytes is available after the framing at the link layer, rely on various addressing and security option.

Interest Packet

\begin{tabular}{|c|}
\hline Content Name/ Prefix \\
\hline $\begin{array}{c}\text { Selector } \\
\text { (order preference, publisher filter, } \\
\text { scope,..) }\end{array}$ \\
\hline
\end{tabular}

Data Packet

\begin{tabular}{|c|}
\hline Content Name \\
\hline $\begin{array}{c}\text { Signature } \\
\text { (Digest algorithm, witness,..) }\end{array}$ \\
\hline $\begin{array}{c}\text { Signed Info } \\
\text { (publisher ID, key locator, stale time,..) }\end{array}$ \\
\hline Data \\
\hline
\end{tabular}

Figure 2 CCN message format [5]

\subsubsection{Message exchange}

An IM is broadcasted over the network connectivity with reference to a consumer requests for content in a one-hop node of the multi-hop wireless environment. The nodes receiving a message and having the requested data that matches the Content name or prefix may transmit the corresponding $\mathrm{CO}$ message. An Interest that matches the Data can only be transmitted in reply.

For an individual established Interest message, a node will transfer a response with compulsorily one CO message, even though the node contains various similar COs. This mapping among IMs and Content messages keeps stability in the flow that lets the receiver to manage the speed of the data transferred from a sender. It saves the bandwidth by transferring the data where it is not requested.

Unsatisfied IMs are retransmitted in some fix time interval for a reliable delivery. A timer is maintained at the receiver side for the unsatisfied Interests and to transmit it again when the timer expires.

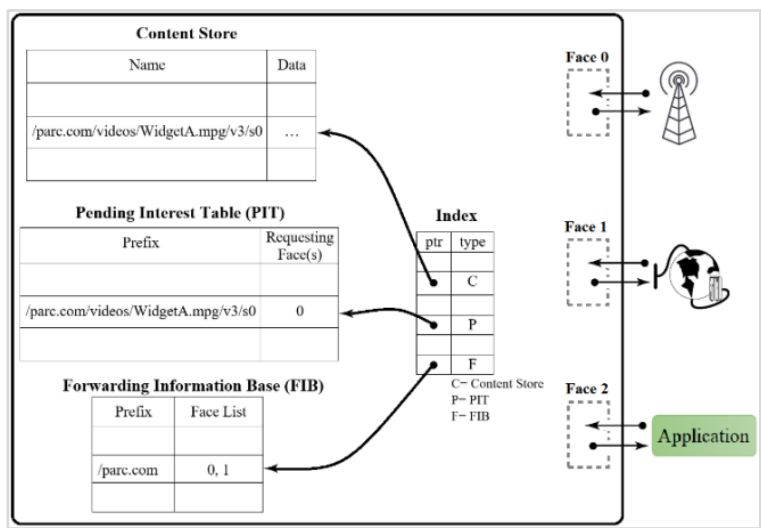

Figure $3 \mathrm{CCN}$ node model [6]

\subsubsection{Node model}

A node model involves following the data structures, according to the specifications of $\mathrm{CCN}$ to offer caching, response to the content requests and to direct messages to different nodes in the network [4]. The index table is a pointer to the data structures table discussed below.

Face: A face is a basic overview of an interface. In the context of specifications of $\mathrm{CCN}$, a face can be a link to a network or it can be a link directly to an application as shown in Figure 3. It can be set up using a tunneling mechanism using P2P (point-topoint) addressing for transmitting and accepting data on a particular network as well as to broadcast messages. In a wireless sensor network, a node comprises of a single network interface controller.

Content Store (CS): The CS can also be called as a cache where data is stored. It also holds the COs formed locally via the information gathered by sensor devices and also the COs acknowledged from other nodes. An index pointer is used to enable content retrieval and suppression. It is not essential to take any distinct procedures to preserve $\mathrm{CO}$ messages in a $\mathrm{CS}$ as it is a cache and not a permanent store. On receiving a different value of data, the data in CS is updated with the current content.

Forwarding Information Base (FIB): A FIB table comprises of a prefix and a face identifiers list as shown in Figure 3. The FIB table is organized to 
retrieve the longest prefix match scheduled on names. A prefix entry in a FIB table is a pointer to a set of destinations instead of a particular one. To retrieve a matching content, a FIB table shows a list of faces that directs its Interest message to a node which is a process equivalent to the IP routing table. A node will direct an Interest to minimum one of the faces whose identifier is involved in the index of FIB table, to retrieve data that matches the prefix.

Pending Interest Table (PIT): PIT is a source table considering the Interests that are not satisfied. It is structured to retrieve a full prefix pairing on names (a match is obtained only when a prefix interest completely matches the prefix of PIT entry) by lookup. PIT entry points to a list of face identifiers which are sources of the undetermined Interest.

As IMs are broadcasted in the network towards possible origin of leaving data, from the intermediary nodes, the PIT entries mark the path of a corresponding data packet that gets back to range the original request for the content. The IMs are routed in $\mathrm{CCN}$, in this manner. Once a node discards the data packet or receives content data which pairs with a pending Interest, a matching PIT entry is forwarded to the corresponding Data packet which is then removed from the table.

PIT entries are maintained temporarily so, it times out after a specific period of time. When pending Interests does not find a corresponding content, it expires and is later removed from the PIT. It depends on the consumer node, to request the Interest message again if it is concerned with receiving the content data. Here we note that the requester of the data preserves the track of Interest messages in its PIT and sends face identifier 0 to use it.

2.3.5Routing and forwarding

In $\mathrm{CCN}$ routing and forwarding the packets can be considered as a smart operation in a wireless environment, routing process can be considered as the way FIB table is filled by interchanging the prefix name by publishing among the routers while Forwarding states that the processing of interest and data that is done hop by hop. Initially, to fill the FIB tables the $\mathrm{CCN}$ nodes can use any traditional routing protocols. It generally depends on flooding-based methods to find the data content in a wireless network. The basic flooding-based methods for data distribution in $\mathrm{CCN}$ can be introduced with some selection of the $\mathrm{CCN}$ forwarding decision process. There are two forwarding approaches blind and aware [7].
In blind forwarding, it mainly supports flooding, which is the simplest method to direct the Interest packets in the wireless network. Some distributed packet suppression techniques can be used as a solution to the packets redundancy and collisions. The blind forwarding does not always ensure that the best nodes are designated to packet forwarding; hence a more advanced, aware forwarding approach was introduced.

In aware forwarding, some awareness mechanisms are included to select the outgoing interface and the next hop nodes based on some selection criteria.

Interface selection is based on the criteria in which forwarding $\mathrm{CCN}$ packets may use the data kept in PITs and FIBs for the selection of outgoing interface(s) at a single node. For instance, the FIB may preserve the path of the delivery performance (in terms of latency, cost) of each interface leaving, to transfer the data packets through the best interface [8].

Next- hop selection where a CCN node can also use some awareness for the selection of subsequent hop in Interest forwarding/ Data forwarding. BlooGo proposal [9] determines whether to forward the packet or not, by comparing the neighbouring of the sender and receiver which can be done using Bloom filter with the data packets.

Path selection is done where the Interest requested by consumer propagates through multiple paths towards the multiple data provider, and also the data packets can return through various paths when several replicas of content data are cached in the intermediate nodes in the network. The diversity of data over various paths is utilized in a VANET [10]. Through some coding techniques of the network, where intermediary nodes make a linear combination of the data established, and transfer the outcome to their neighbouring node.

Provider selection is considered when a consumer gets more than one source for the content requested. The best provider of the content data is selected on some criteria. In [11], the provider selected is advertised with successive Interests that consequently routes the packets to the intermediate nodes. Light path-state information is the provider's identifier selected and the hop distance to the receiving node involved in Interest and Data. That is kept as the fields in a provider table of the $\mathrm{CCN}$ nodes in the wireless ad-hoc network. That gives a dual benefit of 
controlled channel load and low energy consumption.

\section{Discussion on data delivery with $\mathrm{CCR}$ in IoT}

The recent researches in $\mathrm{CCN}$ for IoT are varied in different environments and application area. The $\mathrm{CCN}$ or named data networks (NDN) which aims to caching and subscribing data on the basis of content instead of the host. Also, the data access is independent of location, which enables in-network caching resulting in reduced network traffic $[12,13]$.

MobCCN protocol [14] is proposed for routing and forwarding mechanisms, with their performance evaluation with the different network traffic loads. Different naming and caching schemes for deployment of the $\mathrm{CCN}$ are discussed [15].

Hierarchical naming is used in NDN and CCN approaches. To name the contents, the hierarchical structure is followed which is similar to the URLs in the web pages. With the present Internet applications, Hierarchical naming provides a better compatibility and also provides name aggregation.

The other naming scheme is flat names that are of any fixed size and are easy to operate in routing as it takes fewer resources for computing, and consumes less amount of space while saving. In [16], the flat naming scheme for Wireless networks is proposed in which the naming scheme is divided into two parts: the initial part is used to find category and second is used for content. Also, they explored the CCN naming mechanisms in Contiki OS and the results show the naming mechanism presented performs better than IP in terms of energy consumption and network delay.

In, [17] authors have proposed a hybrid naming scheme for Smart Campus. Hierarchical and Flat components are combined in a Hybrid naming scheme. The naming scheme proposed takes the domain name, location and task as hierarchical component and a hash of device name and data as a flat/hash component. The proposed naming scheme is implemented in Contiki OS.

The evaluation of the execution of $\mathrm{CCN}$ is implemented with data transfer efficiency and content distribution efficiency [6] with the usefulness for both content distribution and point-to-point network protocols that was released as an open source. Design and implementation of stateless multicast RPL forwarding (SMRF) algorithm for IPv6 wireless sensor networks using trickle algorithm is demonstrated with the performance and energy consumption [18-21].

Table 1 provides the comparison of various research projects, evaluated with the naming scheme, architecture and the parameters evaluated with the simulation platform and the programming language used in the implementation.

Table 1 An overview of CCN research projects

\begin{tabular}{lllll}
\hline $\begin{array}{l}\text { Ref. } \\
\text { paper }\end{array}$ & $\begin{array}{l}\text { Naming } \\
\text { scheme }\end{array}$ & Architecture & Parameters evaluated & $\begin{array}{l}\text { Simulator(Os, } \\
\text { Language) }\end{array}$ \\
\hline$[4]$ & Hierarchical & CCNx & Average delay with cache enable \& disable & $\begin{array}{l}\text { Contiki OS and Cooja } \\
\text { Simulator }\end{array}$ \\
{$[19]$} & Hierarchical & CCN & Throughput, success ratio & NS-3 and CCNx \\
{$[20]$} & Hierarchical & CCNx & Latency of CoAP over CCN & $\begin{array}{l}\text { Contiki OS and Cooja } \\
\text { Simulator }\end{array}$ \\
{$[6]$} & Hierarchical & NDN & $\begin{array}{l}\text { Content retrieval time \& data overhead for pedestrian \& } \\
\text { vehicular scenario }\end{array}$ & $\begin{array}{l}\text { NS-3 } \\
\text { Simulator } \\
\text { Contiki OS and Cooja } \\
\text { simulator } \\
\text { C Language }\end{array}$ \\
\hline 16$]$ & Flat Name & CCNx & Average energy consumption & ark \\
\hline
\end{tabular}

\section{Challenges and research solutions for $\mathrm{CCN}$}

In this segment, the issues with the possible solutions for future research directions that need to be solved by researchers are presented briefly.

\subsection{Naming}

Hierarchical naming is generally used in $\mathrm{CCN}$ based IoT naming. Also based on application demand the naming differs. The packet header size is fixed length [23]. The general idea is to show the hierarchy of the naming components that classify the application and the attributes that define the associated contents. The 
$\mathrm{CCN}$ naming is still under dynamic research, and a few naming proposals in context with the wireless networks have appeared as a part of the application domain. Flat names [5] can be obtained by applying hash algorithms to already existing contents and are not applicable to the dynamic contents, whereas hierarchical names provide the request of dynamic IoT contents that are created on demand (e.g., a parameter measured by a sensor), only if the naming conventions are stated in the system configuration.

\subsection{Caching}

Though caching is a distinct feature of $\mathrm{CCN}$ for IoT, it has gained a lot of attention by researchers. It is useful to speed up content retrieval and increase data availability. The cache decision and replacement policies are taken into consideration for various research perspectives [24]. Although caching operations are relatively expensive with respect to processing and bandwidth consumption. Mostly these policies show the issue of usage of space with content popularity. The $\mathrm{CCN}$ based caching schemes also include the freshness value of the content to choose the caching contents. Though the content popularity is also included in the policies it is still required to explore new methods for content popularity [25].

\subsection{Security}

Security is the most demanding research issue that needs to be undertaken in CCN. Most of the CCN security-related solutions are provided for the wired topology and the sensor networks still need to be configured for the security by the research community. The PKI (Public Key Infrastructure) is very complex to implement as it requires more power in the implementation of trust management and key generation in constrained oriented devices [19]. The main drawback in context with key management requires the pre-distribution of keys that creates inflexibility. Usually, the $\mathrm{CCN}$ functions for security should be flexible enough in the choice of different cryptographic methods that can be chosen based on different applications and device capability [5].

\section{Conclusion and future work}

The literature survey provided primary $\mathrm{CCN}$ design keys to fulfill IoT requirements and open research opportunities. But an important question still remains regarding the practical deployment of CCN. Generally, CCN solutions are deployed as an overlay over the current IP network or it can be implemented directly using clean-slate solutions to substitute current IP. Due to the complexity, in the overhead and encapsulation with IP protocol, the overlay solutions are generally not preferred, but it can be used as per the application requirement. A clean slate solution can be deployed easily as there is no requirement of communicating with IP nodes or to preserve backward compatibility. With reference to the design deployment of $\mathrm{CCN}$, lets the coexistence of IP technology that can be facilitated by $\mathrm{CCN}$ hierarchical names that are similar to the URLs of web pages. Hence, we can say that $\mathrm{CCN}$ has the potential to provide a network solution for IoT. In this review paper, we emphasized on the core concepts of CCN and discussed the different methods of routing and packet forwarding techniques. Also, we have discussed and presented a literature survey on CCR techniques and their comparisons to identify the suitability of them under different environment. It has been analyzed that various important parameters such as delay, throughput, latency and energy consumption can be handled efficiently by the appropriate selection of any of the technique. In addition, future work may involve developing an alternate intelligent technique that can further handle above-mentioned parameters efficiently.

\section{Acknowledgment}

None.

\section{Conflicts of interest}

The authors have no conflicts of interest to declare.

\section{References}

[1] http://www.internet-of-things-research.eu/. Accessed 20 August 2018.

[2] Tyson G, Sastry N, Rimac I, Cuevas R, Mauthe A. A survey of mobility in information-centric networks: challenges and research directions. In proceedings of the ACM workshop on emerging name-oriented mobile networking design-architecture, algorithms, and applications 2012 (pp. 1-6). ACM.

[3] Arshad S, Azam MA, Rehmani MH, Loo J. Recent advances in information-centric networking based internet of things (ICN-IoT). IEEE Internet of Things Journal. 2018.

[4] Saadallah B, Lahmadi A, Festor O. CCNx for contiki: implementation details (Doctoral dissertation, INRIA).

[5] Jacobson V, Smetters DK, Thornton JD, Plass MF, Briggs NH, Braynard RL. Networking named content. In proceedings of the international conference on emerging networking experiments and technologies 2009 (pp. 1-12). ACM.

[6] Amadeo M, Campolo C, Molinaro A. A novel hybrid forwarding strategy for content delivery in wireless information-centric networks. Computer Communications. 2017; 109:104-16.

[7] Amadeo M, Campolo C, Molinaro A, Ruggeri G. Content-centric wireless networking: a survey. Computer Networks. 2014; 72:1-13. 
[8] Yi C, Afanasyev A, Moiseenko I, Wang L, Zhang B, Zhang L. A case for stateful forwarding plane. Computer Communications. 2013; 36(7):779-91.

[9] Angius F, Gerla M, Pau G. Bloogo: bloom filter based gossip algorithm for wireless NDN. In proceedings of the ACM workshop on emerging name-oriented mobile networking design-architecture, algorithms, and applications 2012 (pp. 25-30). ACM.

[10] Talebifard P, Leung V. A content centric approach to dissemination of information in vehicular networks. In proceedings of the ACM international symposium on design and analysis of intelligent vehicular networks and applications 2012 (pp. 17-24). ACM.

[11] Amadeo M, Molinaro A, Ruggeri G. E-CHANET: routing, forwarding and transport in informationcentric multihop wireless networks. Computer Communications. 2013; 36(7):792-803.

[12] https://wiki.fd.io/view/Cicn. Accessed 20 August 2018.

[13] Jin Y, Gormus S, Kulkarni P, Sooriyabandara M. Content centric routing in IoT networks and its integration in RPL. Computer Communications. 2016; 89:87-104.

[14] Borgia E, Bruno R, Passarella A. Making opportunistic networks in IoT environments CCNready: a performance evaluation of the MobCCN protocol. Computer Communications. 2018; 123:8196.

[15] Amadeo M, Campolo C, Quevedo J, Corujo D, Molinaro A, Iera A, et al. Information-centric networking for the internet of things: challenges and opportunities. IEEE Network. 2016; 30(2):92-100.

[16] Dinh NT, Kim Y. Potential of information-centric wireless sensor and actor networking. In international conference on computing, management and telecommunications 2013 (pp. 163-8). IEEE.

[17] Arshad S, Shahzaad B, Azam MA, Loo J, Ahmed SH, Aslam S. Hierarchical and flat-based hybrid naming scheme in content-centric networks of things. IEEE Internet of Things Journal. 2018; 5(2):1070-80.

[18] Oikonomou G, Phillips I, Tryfonas T. IPv6 multicast forwarding in RPL-based wireless sensor networks. Wireless Personal Communications. 2013; 73(3):1089-116.

[19] Bosunia MR, Kim A, Jeong DP, Park C, Jeong SH. Efficient data delivery based on content-centric networking. In international conference on big data and smart computing 2014 (pp. 300-4). IEEE.

[20] Subramanian SS, Pasquale J, Polyzos GC. CoAP for content-centric networks. In consumer communications \& networking conference 2017 (pp. 467-72). IEEE.

[21] Yick J, Mukherjee B, Ghosal D. Wireless sensor network survey. Computer Networks. 2008; 52(12):2292-330.

[22] Waltari O, Kangasharju J. Content-centric networking in the internet of things. In consumer communications $\&$ networking conference 2016 (pp. 73-8). IEEE.
[23] http://named-data.net/doc/ndn-tlv/tlv.html. Accessed 20 August 2018.

[24] Quevedo J, Corujo D, Aguiar R. Consumer driven information freshness approach for content centric networking. In conference on computer communications workshops 2014 (pp. 482-7). IEEE.

[25] Hail MA, Amadeo M, Molinaro A, Fischer S. On the performance of caching and forwarding in information-centric networking for the IoT. In international conference on wired/wireless internet communication 2015 (pp. 313-26). Springer, Cham.

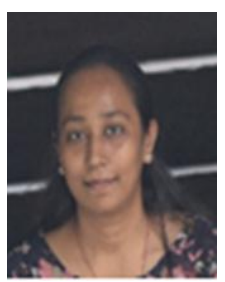

Miss. Pooja Patel is currently pursuing her Post graduation in computer engineering from LDRP institute of technology, affiliated under Kadi Sarva Vidyalaya, India. She has completed her Bachelor of Engineering from Computer Engineering from Sankalchand Patel College of Engineering (SPCE) in year 2013. Few of her domains of interest are Internet of Things (IoT), Sensor networks and Cloud computing Email: prpate19110@gmail.com

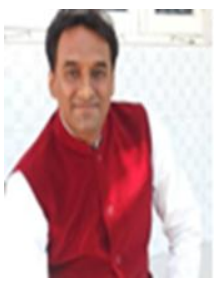

Mr. Hiren Patel is currently working as a Professor and Head in Computer Engineering Department of LDRPInstitute of Technology and ResearchGandhinagar, India. He completed his $\mathrm{Ph} . \mathrm{D}$. from National Institute of Technology (NIT), Surat with Cloud Computing as the domain of research. He completed his post-graduation from M. S. University, Baroda and graduation from S. P. University, V.V.Nagar. Having more than 15 years of teaching experience, Dr. Patel has many research papers in various conferences \& journals of international repute. Few of his main subjects of interest are Computer Programming, Cloud computing, Parallel Processing, Computer Networking and Information Security and Block Chain Technology.

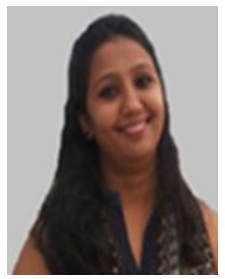

Mrs. Bela Shrimali is currently working as an Assistant Professor in Computer Engineering Department of LDRP-institute of technology and research-Gandhinagar, India. She has completed her Ph.D in Cloud computing domain from C.U. Shah university and masters in computer science and engineering from Government engineering college, Gandhinagar-India. She is having more than 12 years of teaching experience. Ms. Shrimali has presented and published many research papers in various conferences $\&$ journals of international/national repute. Few of her main subjects of interest are Cloud computing, Wireless sensor networks and Blockchain technology. 